

\section{Does personality play a role in continuous positive airway pressure compliance?}

Obstructive sleep apnoea (OSA) is a condition characterised by repetitive, intermittent partial or complete collapse/obstruction of the upper airway during sleep. Continuous positive airway pressure (CPAP) is highly efficacious in treating OSA but its effectiveness is limited due to suboptimal acceptance and adherence rates, with as many as $50 \%$ of OSA patients discontinuing CPAP treatment within the first year. Until recently, research has focused on examining mechanistic and demographic factors that could explain nonadherence (e.g. age, sex, race and education level) with limited applicability in a prospective or clinical manner.

More recent research has focused on personality factors or types of patients with OSA who comply and do not comply with CPAP adherence in an attempt to enhance the accuracy of predicting treatment compliance. Type $D$ personality has been found to be prevalent in one third of patients with OSA. The presence of Type D personality increases noncompliance and poor treatment outcomes due to negative affectivity, social inhibition, unhealthy lifestyle, and a reluctance to consult and/or follow medical advice. Conversely, individuals who are more likely to adhere to CPAP treatment tend to have a high internal locus of control and high self-efficacy, self-refer for treatment, and have active coping skills. By assessing personality and coping skills, the clinician may gain insight into the likelihood of a patient's adherence to treatment. If the patient displays potential risk factors for CPAP noncompliance, the clinician can offer the patient education, refer them to a support group, engage in behavioural/motivational therapy and undertake regular follow-up visits or phone calls incorporating troubleshooting to increase CPAP adherence, especially in individuals with Type D personality.

\section{@ ERSpublications}

OSA patients should be assessed for Type D personality to determine likely CPAP compliance http://ow.ly/Dlbb309cMPH
Cite as: Maschauer EL, Fairley DM, Riha RL. Does personality play a role in continuous positive airway pressure compliance? Breathe 2017; 13: 32-43.
Obstructive sleep apnoea (OSA) is a condition characterised by repetitive, intermittent partial or complete collapse/obstruction of the upper airway during sleep. During wakefulness, dilator muscles contract during inspiration to maintain airway patency. This prevents collapse as a consequence of suction during inspiration. However, during sleep, the tone of these muscles falls, causing narrowing or total obstruction of the airway.

Continuous positive airway pressure (CPAP) is highly efficacious in treating OSA but its effectiveness is limited due to suboptimal acceptance and adherence rates; $5-89 \%$ of patients will reject CPAP as a treatment immediately, $25-50 \%$ of patients who 
commence treatment will fail to continue [1-4], 50\% will discontinue CPAP treatment within 1 year [5] and $25 \%$ terminate CPAP treatment within 3 years [1] This large prevalence of nonadherence decreases its effectiveness, and is limited in improving daytime sleepiness, mental and physical health, and cognitive functioning $[1,6]$. These nonadherence numbers are similar to other populations with health concerns, where roughly $20-40 \%$ of individuals with acute illness, 30-60\% with chronic illness and 50-80\% of those using preventative care are nonadherent to their prescribed medical treatment [5].

Research has focused on finding common qualities and/or circumstances that may lead to nonadherence, such as age, sex, race, apnoea hypopnea index $(\mathrm{AHI})$, body mass index $(\mathrm{BMI})$ and education levels, but these have been inconclusive, equivocal or confounded by other demographic variables [1, 4]. Therefore, more recent research has focused on the psychosocial and personality traits that may contribute to noncompliance, shifting the focus of patient treatment from socioeconomic, physical and environmental predictors to that of more stable personality factors [1, 2, 5].

AIKENs et al. [7] evaluated patients with OSA using the Minnesota Multiphasic Personality Inventory (MMPI), and discovered that $58 \%$ of these patients demonstrated at least one MMPI elevation and 38\% had two or more, with depression, hypochondriasis and hysteria being the highest. From this study, it was reported that those with core depressive symptoms on the MMPI had less severe OSA, and those with a diverse set of psychological symptoms stronger than depression had a greater $\mathrm{AHI}$ and lower oxygen saturation [7]. Depressive symptoms and anxiety are often seen in patients with OSA, which worsens fatigue, but CPAP decreases fatigue providing significant improvement in depression and anxiety [8]. OSA patient satisfaction with CPAP treatment positively correlates with adherence, with the greater the day to day improvement noticed the greater the likelihood of using CPAP [9].

\section{Methods}

\section{Method}

We conducted a review of published, peer-reviewed articles on personality factors that increase risk of nonadherence to CPAP therapy.

\section{Search strategy}

The databases used to search the literature for this review were MEDLINE (2000-present), EMBASE (2000-present), PsycINFO (2000-present), SCOPUS (2000-present) and PubMed (2000present). These databases allowed for a wide range of clinical medical material to be covered over a broad base of global journals. Each database was searched between August and November
2016. Recommendations from the Cochrane Collaboration for a comprehensive, sensitive and wide-variety search were followed to ensure all the highest standards in evidence-based research were undertaken and all relevant articles for this review were identified for review [10]. No conflicts of interest were identified. The following search terms were used; ([CPAP] OR [continuous positive airway pressure]) AND [(OSA) OR (obstructive sleep apnoea or obstructive sleep apnea)] AND (personality)] AND [(non-compliance or compliance]. The additional limit was "to all adult (plus 18 years)" (table 1 ).

\section{Study selection}

All titles and abstracts were assessed and full texts of the relevant studies were obtained if they fulfilled the required inclusion criteria (see later). Selected publications were assessed by two reviewers (E.L. Maschauer and D. Fairley) separately to reduce selection bias. Once a list of articles was created that each reviewer felt met the inclusion criteria, they met to compare results and discuss which articles would be included in the final review.

\section{Study type}

The inclusion criteria were that the studies had to investigate personality types, factors or traits in individuals with OSA and compliant/noncompliant CPAP use. All studies that did not meet the criteria or were not peer-reviewed, published articles were not included in the review. Duplicate studies and those that were not in English were excluded.

\section{Study group}

The study group had to have consisted of adults over the age of 18 years with OSA on CPAP.

\section{Date of Publication}

Studies published between 2000 and 2016 were eligible.

This search identified a total of 13 studies to be included in the review (table 1). The measures used to assess personality factors in these articles consisted of the:

- MMPI, which scores indicators of personality characteristics [11, 12];

- behavioural inhibition system/behavioural activation system (BIS/BAS) questionnaires exploring appetitive and aversive motives [4];

- Type D scale (DS14 and DS16) to assess for Type D personality [3, 6, 9];

- Big Five, NEO Five Factor Inventory and MiniInternational Personality Item Pool, which all measure the five main domains of personality (neuroticism, extraversion, openness, agreeableness and conscientiousness) [4, 9]; 


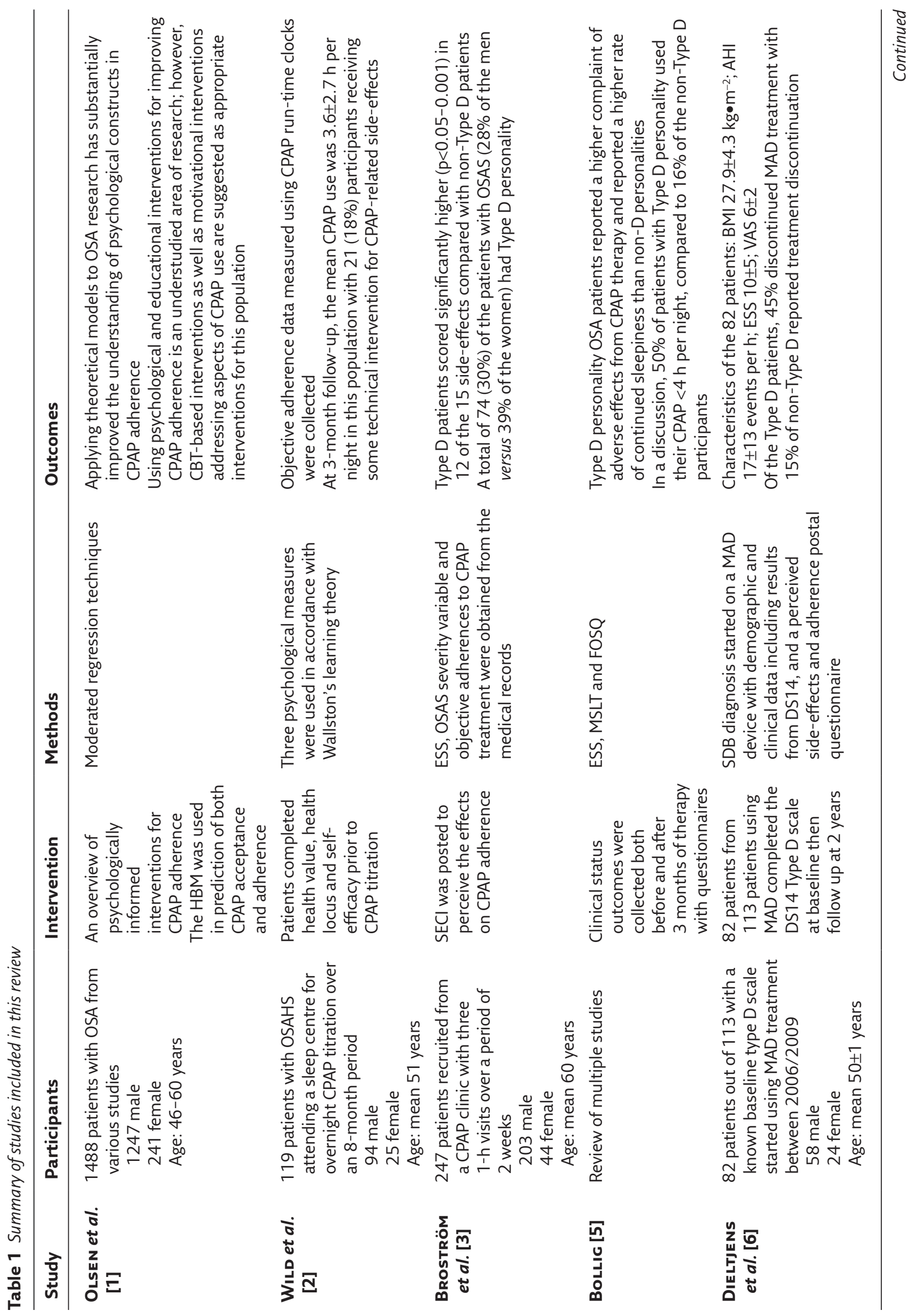




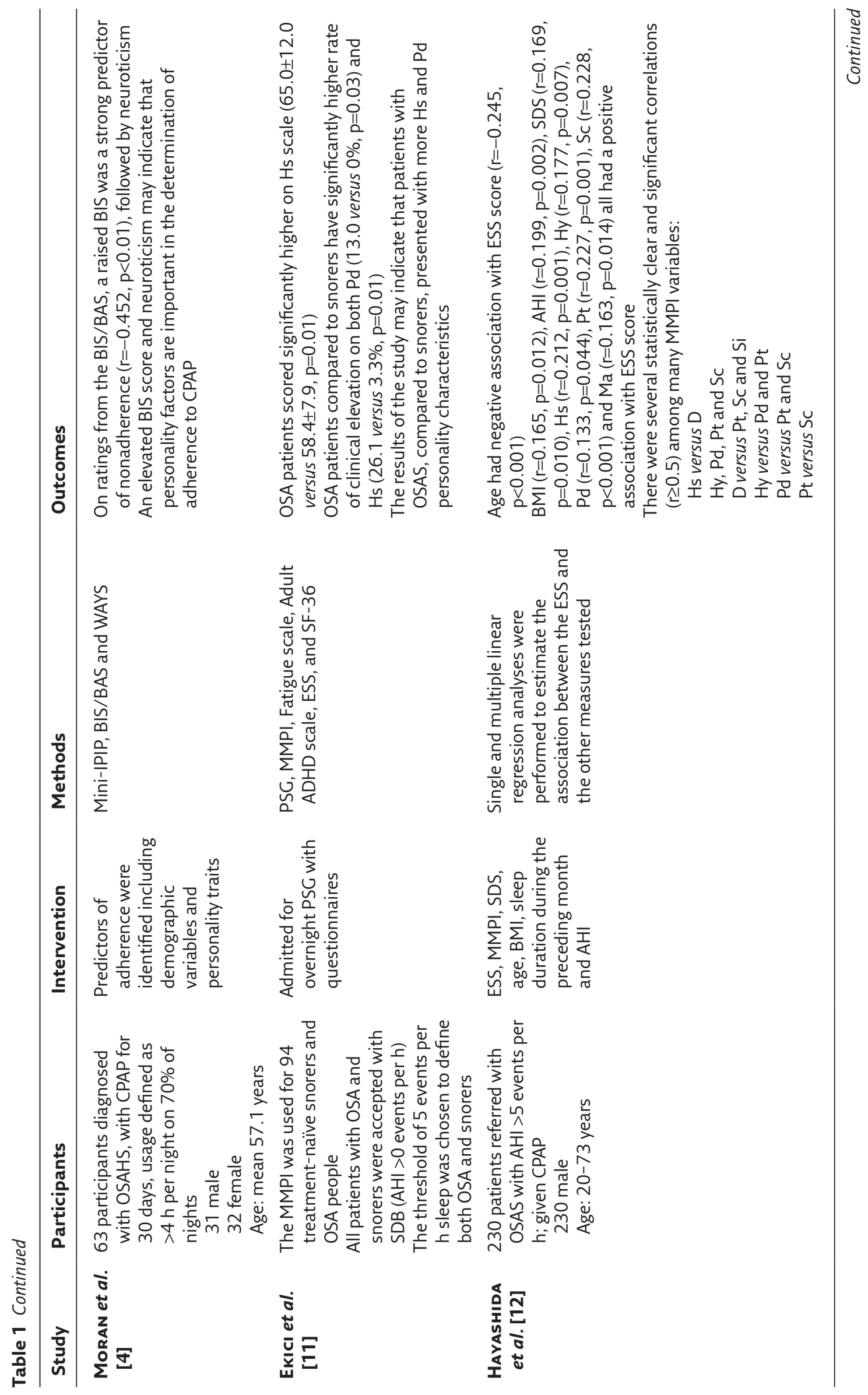




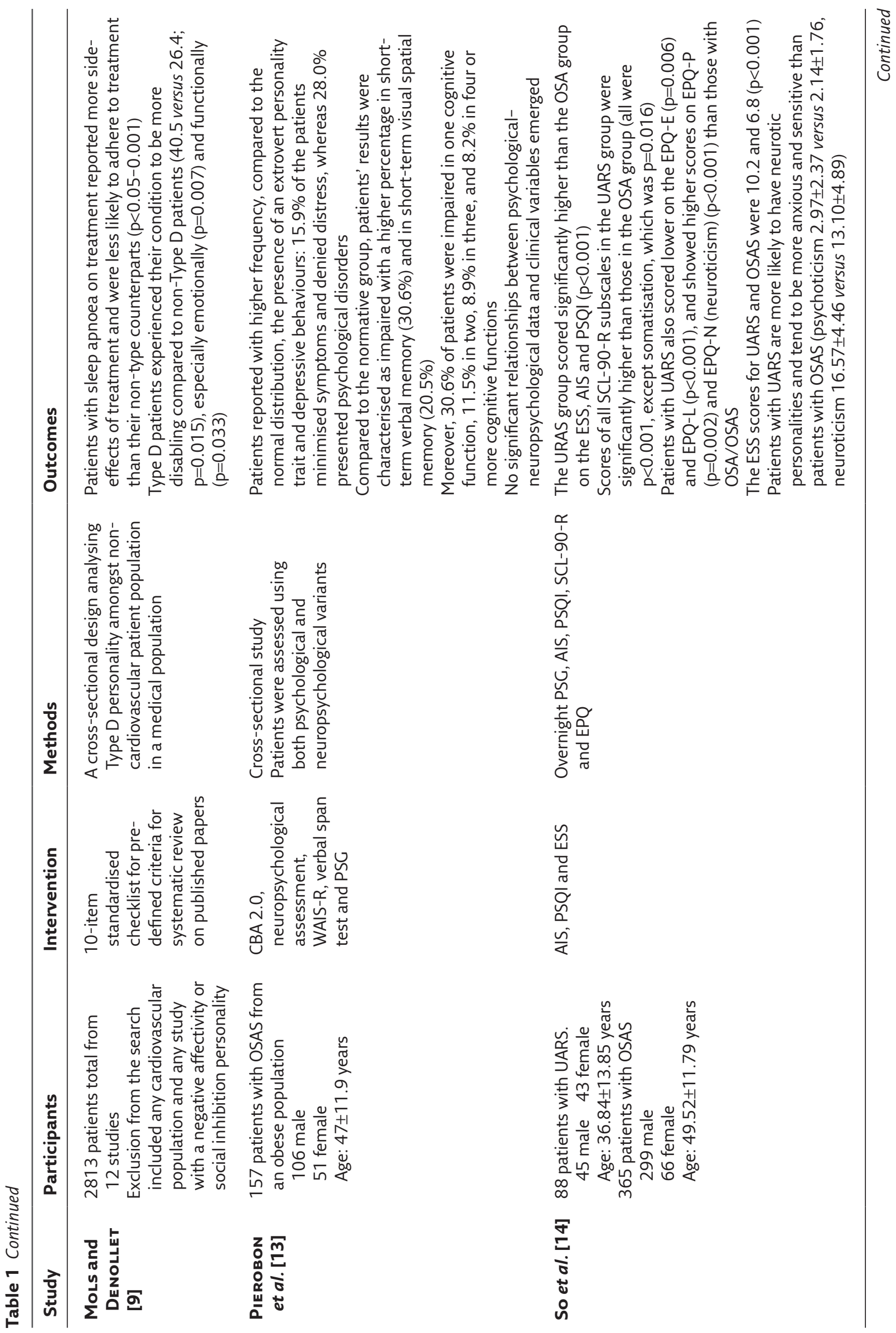



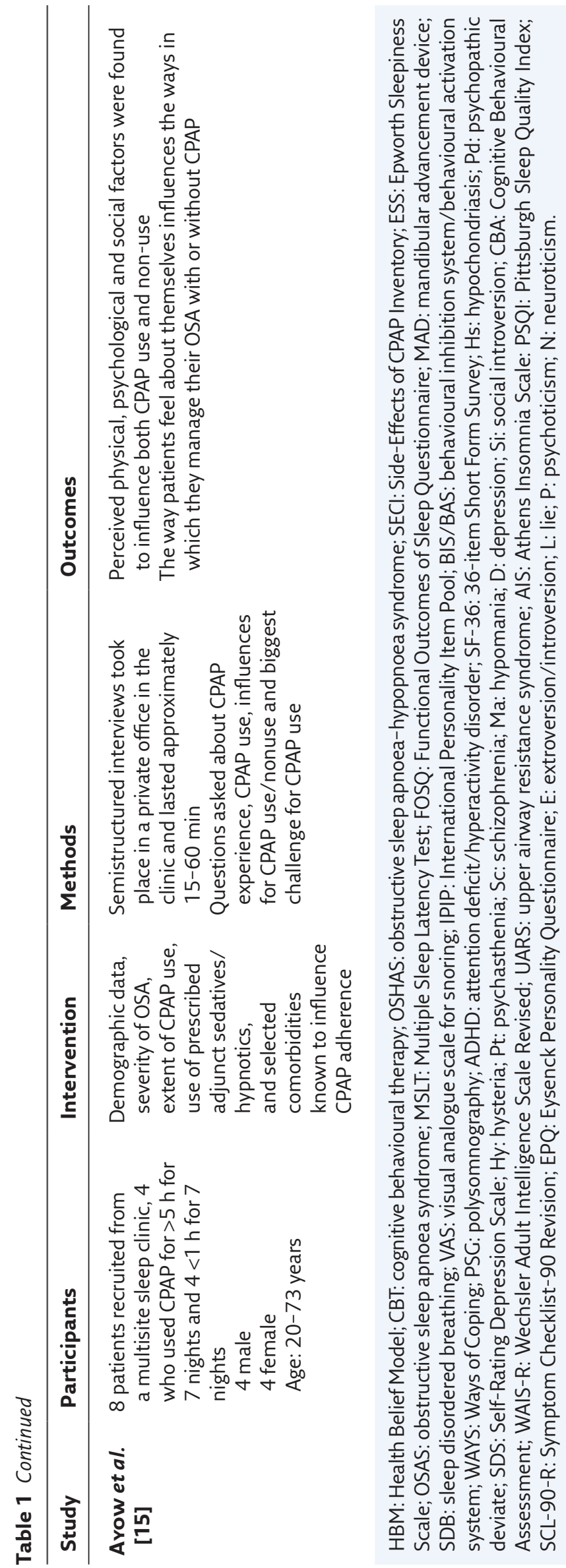

- DOCCO to evaluate stress, personality and lifestyle [4];

- Cognitive Behavioural Assessment, evaluating state and trait anxiety, personality characteristics, psychophysiological disorders, fears and phobias, and depressive behaviours [13];

- Ways of Coping, measuring different domains or aspects of personality, including basic descriptive traits, motivation and coping skills [4];

- Symptom Checklist-90 Revision for psychiatric symptoms [14]; and

- Eysenck Personality Questionnaire to assess personality traits measuring psychoticism, neuroticism, extraversion and lying [14] (table 2).

\section{Personality traits of treatment noncompliers}

Several studies have used personality type indicator measures (e.g. MMPI and Big Five) to investigate individuals with varying degrees of OSA. Type D personality appears to be prevalent in $30 \%$ of the OSA population, in contrast to only $13-24 \%$ in the general population $[3,5,6,9]$ according to several studies conducted in the USA, Sweden, Belgium, Canada, Germany and the Netherlands. Type D personality is characterised by two traits:

- negative affectivity, which is the tendency to experience negative emotions; and

- social inhibition, the hindering of emotional and behavioural expression for fear of rejection or disapproval by others [4-6, 9].

Type $\mathrm{D}$ is a predictor of poor health status and increased risk of mortality in other medical conditions (e.g. chronic pain, mild traumatic brain injury and asthma) [9]. Type D has been linked to certain negative behaviours such as an unhealthy lifestyle, reluctance to consult or follow medical advice, and poor treatment outcomes, adversely affecting the clinical course of medical conditions and treatment compliance [9]. Type D personalities are more likely to be anxious, depressed, socially inhibited, have a decreased quality of life and suffer increased psychological distress [1, 3, $6,9,11]$. OSA patients with Type D personality have poor medication compliance, and lower adherence to CPAP and mandibular advancement devices (MADs) than those who are not Type $D$ $[3,6]$. Roughly $50 \%$ of Type D OSA patients use their CPAP for $<4 \mathrm{~h}$ per night, unlike their nonType D counterparts who use their CPAP for $6 \mathrm{~h}$ a night; $45 \%$ also discontinue MAD treatment $[3,5,6]$. Type $D$ personalities are more likely to have medical comorbidities, a decreased personal view of their own health, decreased physical functioning and poor psychosocial functioning [9]. Their subjective perception of the problem does not always adequately reflect the actual severity of the condition, and they report side-effects of CPAP 


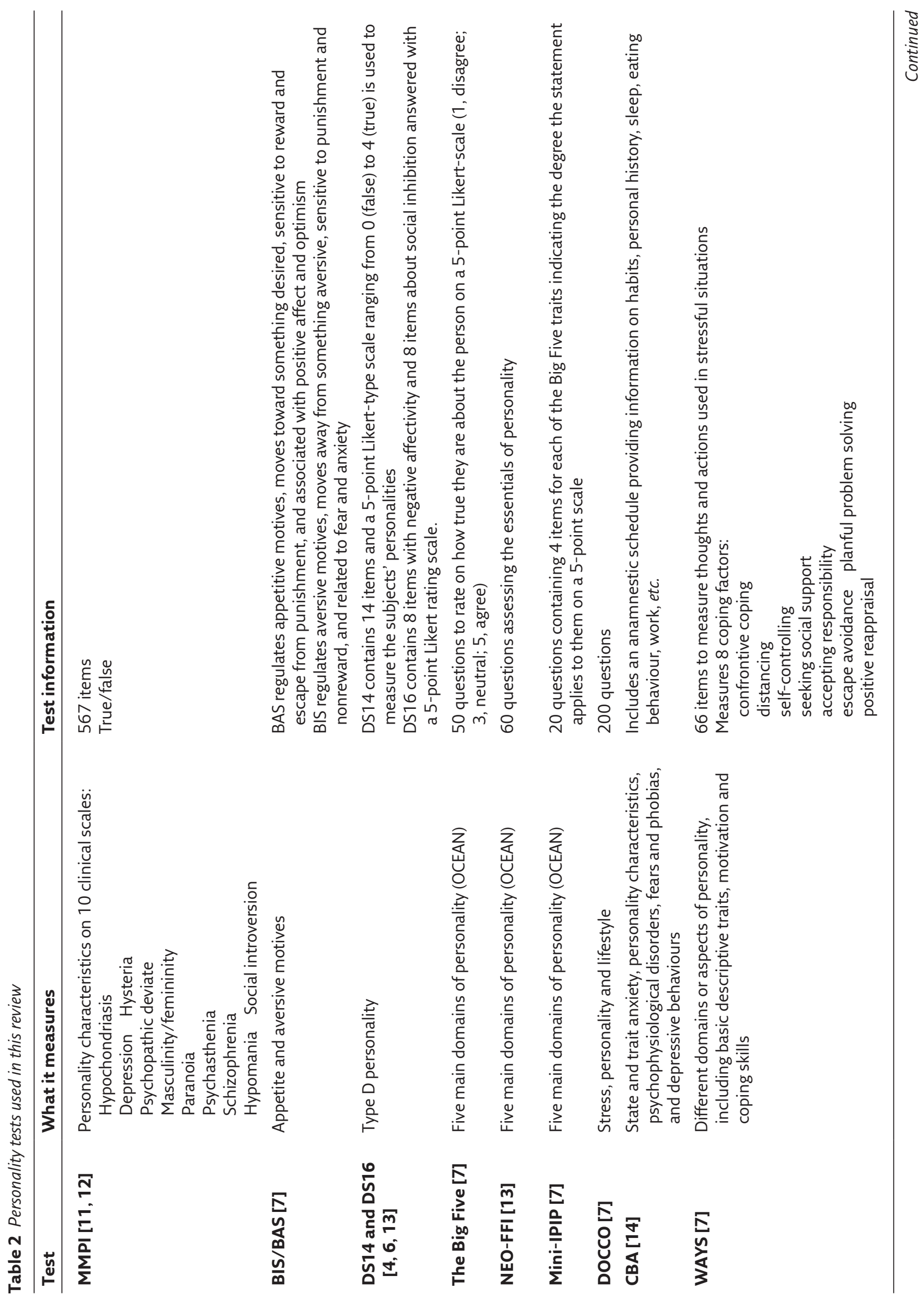




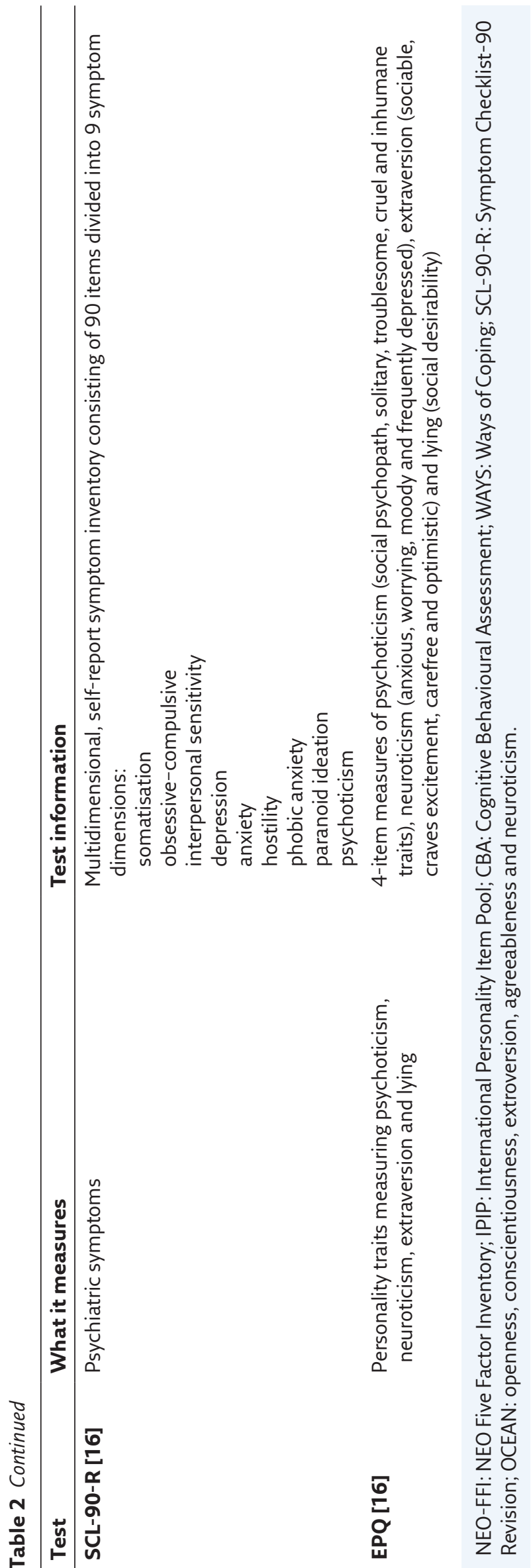

more frequently (e.g. dry throat and mask leaks) [1, $3,9]$. Type D decreased adherence to CPAP may also be caused by decreased perceived effects of the treatment and low self-efficacy [3].

When compared to those with snoring, OSA patients have significantly higher absolute scores on the hypochondriasis, psychopathic deviance and depression scales of the MMPI $[1,11]$. Scores high on the depression and hypochondriasis scales also predict low CPAP adherence [16]. The hypochondriasis scale measures an overconcern with one's state of health [4] and high scores indicate extreme attention to physical symptoms, chronic fatigue and weakness, and noticing vague symptoms, which lead to higher rates of noncompliance [11]. High scores on the hypochondriasis scale also influence subjective daytime sleepiness [12]. High scores on psychopathic deviance may show difficulty adhering to rules and following the advice of authority figures/medical staff, and failing to learn from past mistakes, again increasing risk of continual nonadherence [11]. The prevalence rate of depression in OSA compared to people without OSA is roughly $33 \%$, which may be a direct consequence of sleep deprivation or could be an indirect consequence of social effects caused by the disorder [13], as long-term sleep deprivation can adversely affect mood [3]. Individuals with OSA tend to have a higher BMI than the normative population [15] and obesity is associated with an increased risk of depression as well [13].

The personality patterns of OSA patients with low CPAP adherence have been found to be a somatic-neurotic type, consisting of physical symptoms that derive from the psyche more than from a physical illness [11]. This increased somatic development of symptoms creates a high level of psychological distress for the patient, as a consequence of OSA, not as a cause of it [11]. Other factors that increase low adherence to CPAP are OSA patients who exhibit a significantly high level of denial of disease severity, hysterical personality characteristics, decreased physical activity, feelings of guilt, a pessimistic outlook on life and low selfesteem [11]. Individuals with Type D score high on neuroticism, low on extraversion and low on conscientiousness when evaluated using the Big Five personality model, and score high on the BIS, displaying avoidance and aversion to unpleasant stimuli $[4,9]$. The higher scores on the BIS are possibly due to fear and anxiety developing with respect to CPAP being an invasive and undeniable long-term treatment [4].

Another factor that could give insight into whether a patient will have low CPAP adherence is knowing who scheduled the patient for treatment. If the patient's partner refers them, rather than the patient referring themselves, adherence to CPAP decreases [2]. This phenomenon may be due to Type $D$ personalities having a high external locus of control (e.g. believing their health is determined by external forces, not the internal self) or minimising 
their symptoms, thereby not considering treatment to be necessary $[1-3,15]$.

\section{Personality traits of treatment compliance}

Patients who have a high internal locus of control (e.g. believing they have control over their health) are more likely to adhere to CPAP treatment [1-3, 15]. This is due to internalising and following advice received from sleep specialists, having the ability not to perseverate on treatment side-effects, and displaying high self-efficacy [2]. High self-efficacy empowers individuals to believe in their own ability to deal with obstacles and take control of their own health [5], thus increasing treatment compliance. This also explains why self-referring patients are more likely to adhere to treatment.

Individuals with a high level of conscientiousness and openness, active coping skills, and problem solving traits are also most likely to adhere to treatment [4]. Implementation of coping strategies/ skills in demanding situations can lead to a higher overall level of CPAP use per night and continued use over time [2].

CPAP adherence is also increased if a patient is educated on the negative health outcomes that will develop as a result of not using the treatment, as well as believing they have a more severe form of the disorder [2]. Physiological measures of disease severity rarely accurately predict $>10 \%$ of the variance in adherence [1] but the patient's belief that his/her OSA is severe will lead to higher treatment compliance.

Those who comply more with CPAP tend to have a higher BMI, report less daytime sleepiness, report better nocturnal sleep quality, and score lower on the depression and hypochondriasis scales on the MMPI prior to treatment [16]. These five predictors identify $\sim 80 \%$ of individuals who eventually fail to comply with CPAP treatment as well as $97 \%$ of those who comply long-term [16].

\section{Discussion}

Despite CPAP being an effective treatment for OSA, improving quality of sleep, excessive daytime sleepiness (EDS) and road accidents, patient adherence to this device is relatively low. By better understanding which factors play into the prediction of treatment noncompliance, health professionals can create preventative plans and support systems for patients to increase adherence. One way to increase CPAP adherence is for clinicians to use a short personality inventory (DS14 is recommended [9]) and a coping skills questionnaire to evaluate OSA patients. From this information, clinicians can determine if the patient has a Type $\mathrm{D}$ personality and assess active coping skills,

\section{Educational questions}

1. What are the characteristics of Type D personality?

a) Relaxed, easy going and prone to few health concerns

b) Usually stressed, negative emotions, social inhibition and fear of rejection/disapproval of others

c) Highly strung, organised and unwilling to change or adapt to new situations

d) A mix between Type A (organised) and Type B (relaxed)

2. What are the characteristics of those who are compliant with continuous positive airway pressure (CPAP)?

a) Relaxed, easy going and prone to few health concerns

b) Usually stressed, unable to cope with everyday problems and having an overly involved partner

c) Negative emotions, social inhibition and introverted

d) High internal locus of control, high self-efficacy and active coping skills

3. What can specialists/doctors do to increase CPAP adherence?

a) Wait 4 weeks after giving CPAP and check with the patient

b) Use a personality inventory, coping skills questionnaire, educational programmes/sessions and giving information booklets

c) Stress the importance of CPAP but if a patient is not going to use the machine, there is little that can be done

d) Introduce a CPAP compliance smartphone app into the patient's care plan 4. How can partners affect CPAP adherence?

a) Supporting/reinforcing the patient's desire to use CPAP increases adherence

b) Partners who complain about the noise the CPAP machine emits decrease CPAP adherence

c) Partners who come to the doctor's appointments with the patient increase CPAP asherence

d) Partners who make doctor's appointments for the patient decrease CPAP adherence

whilst the patient awaits CPAP titration. Screening patients before treatment may allow clinicians the opportunity of prescribing a structured, welldesigned intervention that is focused on improving the patient's self-management, coping skills and self-efficacy, thus improving adherence and longterm continuation of CPAP [3, 6].

Assessing for anxiety and depression pretreatment has been found to strongly predict CPAP adherence and may lead to better subsequent CPAP use by educating the patient on how CPAP decreases depression and anxiety [1, 4, 5]. The goal of the CPAP titration appointment should not simply be about treating OSA but should include education on OSA and CPAP, enhance patient acceptance of the treatment, and provide continual support and education, encouraging long-term adherence [1].

Intense educational programmes, mask fitting and a familiarisation session with CPAP prior to CPAP titration also increase adherence $[2,3,5,17,18]$. Training by a sleep specialist or nurse coupled with a video depicting information on OSA, symptoms, health consequences and 


\section{Suggested answers}

1. b.

2. d.

3. b.

4. d. pathophysiology, as well as what a CPAP machine is, how to use it and the benefits of CPAP, should all take place before the patient goes home with the machine. By alerting the patient to the medical and daytime consequences (e.g. weight gain and EDS) of untreated OSA, highlighting the advantages of the treatment (e.g. relationship improvement), downplaying the disadvantages, and stressing the consequences of nonadherence, adherence to CPAP actually increases [6]. Patients should be given a take-home booklet with this information to refer to as necessary and given ongoing education after each clinical visit. There is evidence that providing literature that outlines the value and importance of regular CPAP use increases nightly use by 2.7 more hours a night [1].

If the patient has a Type $D$ personality, early intervention within the first few days of commencing CPAP therapy using cognitive behavioural therapy (CBT), Motivational Enhancement Therapy or motivational interviewing intervention provides coping skills, improves self-efficacy and provides a positive view of the treatment $[17,19,20]$. Type D patients do not effectively cope with stressful life events; therefore, psychological interventions (e.g. CBT) focusing on training and teaching coping skills to decrease stress and increase disease management skills not only benefit the patient, but also increase CPAP use by 2.7-3.2 h a night $[1,5,9]$. Social support groups improve adherence by increasing positive emotions associated with treatment, peer motivation to continue using CPAP long-term [1, 4, 5] and provide coping skills/selfmanagement tools among peers [5].

An important aspect of predicting compliance is to evaluate the perception of symptoms and improvement of those with Type D since these individuals tend to perseverate on the perception of symptoms or side-effects [5]. Therefore, another critical component to increasing adherence is providing nursing support and follow-up phone calls, particularly during the first month of CPAP use $[18,21]$. These troubleshooting telephone calls have a positive impact, increasing CPAP use by $>1.4 \mathrm{~h}$ a night [1]. Telemonitoring (e.g. talking with the patient via Skype instead of in person) also improves CPAP adherence and decreases depressive symptoms [22]. This support, coupled with patient visits to the sleep centre will provide immediate troubleshooting as well as detect early patterns of CPAP use, allowing further intervention if necessary $[1,5,18,21]$. It is recommended these follow-up phone calls and clinical visits should be within the first few days after treatment has commenced, and then again after 6 weeks, 3 months, 6 months and then yearly [5]. Well-designed patient-educational and support programmes coupled with follow-up visits and phone calls can lead to superior adherence of $84 \%$ with $\geq 4$ h a night $[5,23]$.

Lastly, enlisting the support of a significant other is a potential factor that can increase CPAP adherence [1, 5], as CPAP use positively affects relationship quality. Partners should be encouraged to allow the patient to make their own appointments, as adherence increases when patients feel they have a sense of self-control over their disease. While personality type is an important factor to consider in the management of OSA patients, interventions focusing on lifestyle (diet and exercise) could also improve mood, wellbeing and, potentially, CPAP compliance [24]. A comprehensive strategy should be aimed for in all OSA patients.

Most of the studies to date on CPAP adherence and personality have used small sample sizes or are case studies, and have not investigated Type A (organised, anxious) or Type B (relaxed, creative) personality types in this population. CPAP treatment compliance is imperative to decrease mortality rates, decrease economic costs, and increase the quality of life of individuals with OSA and their families. There is a need for more research in understanding and targeting interventions aimed at psychosocial predictors of CPAP adherence, and developing better, more effective and bespoke treatment plans. Since personality traits are not stable and can transform over time after use of an intervention or positive experiences, more research is warranted focusing on cognitive variables $[2,5,11]$. For example, there have not been any studies investigating the change in MMPI scores after CPAP use has been initiated and maintained. Further investigation is necessary to understand how health professionals can increase CPAP adherence and empower patients to deal with their own disease.

\section{Conflict of interest}

None declared.

\section{References}

1. Olsen S, Smith S, Oei TP. Adherence to continuous positive airway pressure therapy in obstructive sleep apnoea sufferers: a theoretical approach to treatment adherence and intervention. Clin Psychol Rev 2008; 28: 1355-1371.

2. Wild MR, Engleman HM, Douglas NJ, et al. Can psychological factors help us to determine adherence to CPAP? A prospective study. Eur RespirJ 2004; 24: 461-465.
3. Broström A, Strömberg A, Mårtensson J, et al. Association of Type D personality to perceived side effects and adherence in CPAP-treated patients with OSAS. J Sleep Res 2007; 16: 439-447.

4. Moran AM, Everhart DE, Davis CE, et al. Personality correlates of adherence with continuous positive airway pressure (CPAP). Sleep Breath 2011; 15: 687-694. 
5. Bollig SM. Encouraging CPAP adherence: it is everyone's job. Respir Care 2010; 55: 1230-1239.

6. Dieltjens M, Vanderveken OM, Van den Bosch D, et al. Impact of type $D$ personality on adherence to oral appliance therapy for sleep-disordered breathing. Sleep Breath 2013; 17: 985-991.

7. Aikens JE, Caruana-Montaldo B, Vanable PA, et al. MMPI correlates of sleep and respiratory disturbance in obstructive sleep apnea. Sleep 1999; 22: 362-369.

8. Habukawa M, Uchimura N, Kakuma T, et al. Effect of CPAP treatment on residual depressive symptoms in patients with major depression and coexisting sleep apnea: contribution of daytime sleepiness to residual depressive symptoms. Sleep Med 2010; 11: 552-557.

9. Mols F, Denollet J. Type D personality among noncardiovascular patient populations: A systematic review. Gen Hosp Psychiatry 2010; 32: 66-72.

10. Lefebvre C, Manheimer E, Glanville J. Searching for studies. In: Higgins J, Green S, eds. Cochrane Handbook for Systematic Reviews of Interventions. Chichester, The Cochrane Collaboration, 2008

11. Ekici A, Ekici M, Oğuztürk O, et al. Personality profiles in patients with obstructive sleep apnea. Sleep Breath 2013; 17: 305-310.

12. Hayashida K, Inoue Y, Chiba S, et al. Factors influencing subjective sleepiness in patients with obstructive sleep apnea syndrome. Psychiatry Clin Neurosci 2007; 61: 558-563.

13. Pierobon A, Giardini A, Fanfulla F, et al. A multidimensional assessment of obese patients with obstructive sleep apnoea syndrome (OSAS): a study of psychological, neuropsychological and clinical relationships in a disabling multifaceted disease. Sleep Med 2008; 9: 882-889.

14. So SJ, Lee HJ, Kang SG, et al. A comparison of personality characteristics and psychiatric symptomatology between upper airway resistance syndrome and obstructive sleep apnea syndrome. Psychiatry Investig 2015; 12: 183-189.
15. Ayow TM, Paquet F, Dallaire J, et al. Factors influencing the use and nonuse of continuous positive airway pressure therapy: $A$ comparative case study. Rehabil Nurs 2009; 34: 230-236.

16. Edinger JD, Carwile S, Miller P, et al. Psychological status, syndromatic measures, and compliance with nasal CPAP therapy for sleep apnea. Percept Mot Skills 1994; 78: 1116-1118.

17. Wozniak DR, Lasserson TJ, Smith I. Educational, supportive and behavioural interventions to improve usage of continuous positive airway pressure machines in adults with obstructive sleep apnoea. Cochrane Database Syst Rev 2014; 8: CD007736.

18. Hoy CJ, Vennelle M, Kingshott RN, et al. Can intensive support improve continuous positive airway pressure use in patients with the sleep apnea/hypopnea syndrome? Am J Respir Crit Care Med 1999; 159: 1096-1100.

19. Aloia MS, Arnedt JT, Strand M, et al. Motivational enhancement to improve adherence to positive airway pressure in patients with obstructive sleep apnea: a randomized controlled trial. Sleep 2013; 36: 1655-1662.

20. Olsen S, Smith SS, Oei TP, et al. Motivational interviewing (MINT) improves continuous positive airway pressure (CPAP) acceptance and adherence: a randomized controlled trial. J Consult Clin Psychol 2012; 80: 151-163.

21. Chervini RD, Theut S, Bassetti C, et al. Compliance with nasal CPAP can be improved by simple interventions. Sleep 1997; 20: 284-289.

22. Sparrow D, Aloia M, Demolles DA, et al. A telemedicine intervention to improve adherence to continuous positive airway pressure: a randomised controlled trial. Thorax 2010; 65: 1061-1066.

23. Dickerson SS, Kennedy MC. CPAP devices: encouraging patients with sleep apnea. Rehabil Nurs 2006; 31: 114-122.

24. Thomasouli MA, Brady EM, Davies MJ, et al. The impact of diet and lifestyle management strategies for obstructive sleep apnoea in adults: a systematic review and meta-analysis of randomised controlled trials. Sleep Breath 2013; 17: 925-935. 